\title{
COMUNICAÇÃO DA EQUIPE DE ENFERMAGEM E A RELAÇÃO COM A GERÊNCIA NAS INSTIT UIÇÕES DE SAÚDE
}

\author{
M aria Cláudia dos SAN T OS ${ }^{a}$, Andrea BE RNARDES ${ }^{b}$
}

\section{RESUM 0}

A comunicação deve acontecer constantemente a fim de reger as atividades gerenciais proporcionando informação, motivação e satisfação nos cargos. Tal estudo objetiva analisar as contribuições das pesquisas produzidas acerca da comunicação na gerência de enfermagem, tomando por base as publicações em periódicos nacionais. T rata-se de revisão integ rativa da liter atura, sendo incluídas dissertações, teses e artigos indexados na L iter atura L atino-A mericana e do Caribe em Ciências da Saúde (LILACS), M edical L iterature Analysis and R etrieval Systems Online (M EDLINE) e Banco de Dados Bibliográficos da Universidade de São Paulo (DEDALUS), no período de 1998 até 2008. 0 fortalecimento do processo comunicativo e a garantia de que ele ocorra de for ma clara e eficiente é essencial para a gerência de enfermagem. É por meio desta comunicação eficiente que o enfermeiro garante identificação de problemas individuais e coletivos, podendo relacioná-los com a análise da situação encontrada e direcioná-los para um planejamento de cuidado apropriado e efetivo.

D escritores: Comunicação. Enfermagem: organização \& administração. E quipe de enfermagem.

\section{RESUMEN}

L a comunicación debe ocurrir constantemente, a fin de regir las actividades gerenciales, proporcionando información, motivación y satisfacción en las personas encargadas. E se estudio tiene como objetivo analizar las contribuciones de las investigaciones producidas acerca de la comunicación en la gerencia de enfermería, basada en las publ icaciones en periódi cos brasileños. E sta es una R evisión Integradora de la L iteratura queincluyó disertaciones, tesis y artícul os indexados en los bancos de datos del L iteratura de A mérica L atina y del Caribe en las Ciencias de la Salud (L IL ACS), M edical L iterature Analysis and R etrieval System Online (M E D L IN E) y Base de datos bibliográfica de la U niversidad de São Paulo (DE DA LUS), entre 1998 y 2008. E I for tal ecimiento del proceso comunicativo y la garantía de que ello ocurra deforma clara y eficientees esencial para la gerencia de enfermería. E s por medio de esa comunicación eficiente que el enfermero garantiza la identificación de problemas individuales y colectivos, pudiendo relacionarlos con el análisis de la situación encontrada y direccionarlos hacia una planificación de cuidado apropiado y efectivo.

Descriptores: Comunicación. E nfer mería: organización \& administración. G rupo de enfermería.

Título: Comunicación del equipo de enfermería y su relación con la gerencia en las instituciones de salud.

\section{ABST RACT}

Communication should occur constantly, in order to guide managerial activities, allowing for the necessary job information, motivation and satisfaction. This study aims to analyze the contribution of the research produced on communication in nursing management, considering the literature published in B razilian journals. Integrative $L$ iterature R eview was used, including dissertations, theses and articles index ed in L atin A merican and Caribbean H ealth Sciences (L IL ACS), M edical $L$ iteratureA nal ysis and R etrieval System O nline (M E D L IN E ) and Bibliographic D atabase of theU niversity of São Paulo (DEDALUS), between the years of 1998 and 2008. Strengthening the communication process and guaranteeing that it occurs in a clear and efficient way is essential for nursing management. Through efficient communication, nurses ensure the identification of individual and collective problems, thus making it possible to relate them to the analysis of the found situation and direct them towards a planning of appropriate and effective care.

Descriptors: Communication. N ursing: organization \& administration. N ursing, team.

Title: Communication of the nursing team and its relationship with management in health institutions.

\footnotetext{
${ }^{a} \mathrm{G}$ raduando do oitavo semestre do Curso de Bacharelado em E nfer magem da E scola de E nfer magem de R ibeirão Preto da U niver sidade de São Paulo (E R R / U SP), Ribeirão Preto, São Paulo, Brasil.

${ }^{b}$ D outora em Enfermagem F undamental, Professora da EERP/ U SP, Ribeirão Preto, São Paulo, Brasil.
} 


\section{INT RODUÇÃO}

0 presente estudo visa discutir a importância da comunicação e sua inter-relação com o gerenciamento das instituições de saúde.

Comunicar é o processo de transmitir informações de pessoa para pessoa, através da fala, da escrita, de imagens e sons com o objetivo de gerar conhecimentos $^{(1)}$ e envolve troca e entendimento de informações, utilizando para isso os sistemas simbólicos.

Para o gerenciamento de qualquer organização, o processo comunicativo é fator essencial para garantir que as atividades ocorram de maneira eficiente e eficaz, devendo acontecer constantemente a fim de proporcionar informação e compreensão necessárias à condução das tarefas, e acima de tudo, motivação, cooperação e satisfação nos cargos.

D entro da área da saúde, percebe-se e valoriza-se a importância da comunicação nas relações entre os profissionais e os usuários do sistema, de forma que possibilite 0 entendimento e a satisfação a todos e a harmonia para a instituição.

No que tange à prática profissional do enfermeiro, percebe-se e destaca-se o valor da liderança, pois é através dela que este profissional garante uma boa gerência e melhoria da qualidade da assistência de enfermagem. Para o sucesso desta liderança, a comunicação se torna imprescindível, já que permite ao enfermeiro se aproximar de sua equipe e demais profissionais com o intuito de compreender as atividades executadas, compartilhar idéias e visões, bem como criar interdependências para o desenvolvimento do trabalho através de equipes(2).

É justamente por meio da competência em comunicação que o enfermeiro garante um bom desempenho das suas funções, inclusive gerenciais. 0 fortal ecimento do processo comunicativo e a garantia de que el e ocorra de forma clara e eficiente é essencial na gerência de enfer magem, já que a troca de informações entre serviços, instituições e população é altamente desejada. É por meio desta comunicação eficiente que o enfer meiro garante a identificação de problemas individuais e coletivos na população, podendo então relacioná-los com a análise da situação encontrada e direcioná-los para um planejamento de cuidado apropriado e efetivo.

Para que o processo comunicativo seja aplicado e desenvolvido com qualidade, 0 enfermeiro deve ser criativo, hábil e, especial mente, capaz de ouvir, permitindo aos trabalhadores explorar todo seu potencial na execução de suas atividades. Como resultado, pode ser percebido uma clareza da comunicação e conseqüente entendimento da informação.

0 enfermeiro deve ainda possuir consciência do verbal e do não-verbal(3) nas interações, principalmente em relação a seus pacientes, que muitas vezes utilizam expressões corporais e gestos, para se comunicar e transmitir a mensagem desejada.

Sabe-se que o modo de organização do trabaIho utilizado interfere na qualidade da comunicação entre os profissionais. No caso do M odelo F uncional a comunicação segue a escala hierárquica, é diretiva e visa o cumprimento de ordens e tarefas. E m contrapartida, há o T rabal ho em Equipe, que visa à organização de um trabalho conjunto entre os membros, acarretando com isto uma comunicação mais aberta e lateral, objetivando assim a prestação de uma assistência qualificada ao paciente.

Como a maioria das instituições mantém ainda vigente um modelo baseado nos princípios da A bordagem Clássica da Administração que representa um modelo hierárquico de gestão pautado no poder de mando-subordinação, o processo de comunicação encontra-se precário, ocorrendo sempre vertical mente, no sentido descendente, sob forma de ordem e raramente de orientação ${ }^{(4,5)}$. Com isto, as pessoas não são ouvidas e as decisões se tornam de baixa qualidade, uma vez que são baseadas em poucas informações, geralmente incompletas e incorretas, além de apresentar um fluxo distorcido e demorado(6).

Paradoxal mente, nos modelos gerenciais mais contemporâneos, pautados em estruturas flexíveis, descentralizadas e ligado à responsabilização dos envolvidos, deve-se pensar menos intensivamente em comunicação vertical e, prioritariamente, em comunicação horizontal ou lateral, incentivando tal processo tanto interunidades como intraunidade ${ }^{(7-9)}$.

A ssim o ato de se comunicar fica facilitado e aproxima as interações pessoais, tornando estas mais agradáveis e produtivas. A organização se torna um grande sistema de processamento de informações e amplia-se a possibilidade de se usar melhor as capacidades individuais e setoriais da organização, em virtude da disponibilidade de informações ${ }^{(10)}$. D esta maneira, reforçamos que a qualidade da comunicação no gerenciamento de en- 
fermagem leva à elaboração de um trabalho harmonioso entre a equipe multiprofissional, além do al cance das reais necessidades do cliente.

0 presente artigo tem como objetivo analisar as contribuições das pesquisas produzidas acerca da comunicação na gerência de enfermagem, tomando por base as publicações em periódicos nacionais e de circulação internacional nos últimos dez anos.

\section{PROCEDIMENT O MET OD OLÓGICO}

Para atender aos objetivos deste estudo, utilizou-se a Revisão Integrativa da Literatura segundo os pressupostos de $\mathrm{G}$ anong ${ }^{(11)}$, que tem o propósito de reunir conhecimentos sobre um determinado tópico, uma vez que proporciona aos leitores os antecedentes para a compreensão do conhecimento atual, facilitando 0 acúmulo de conhecimentos ${ }^{(12)}$. Por meio da revisão integrativa da literatura procurou-se responder como ocorre o processo comunicativo entre os membros da equipe de enfermagem e sua relação com a gerência nas instituições de saúde.

Foram incluídos neste estudo os artigos, dissertações e teses publicadas em língua portuguesa, inglesa ou espanhola no período de 1998 até 2008. A ceitaram-se publicações indexadas nas bases de dados da Literatura Latino-Americana e do Caribe em Ciências da Saúde (LI LACS), M edical L iterature Analysis and R etrieval Systems Online (M ED LINE) e Banco de Dados Bibliográficos da U niversidade de São Paulo (DEDALUS), bem como o acervo bibliográfico da sala de leitura "G lete de A Icântara" da Escola de Enfermagem de Ribeirão Preto da U niversidade de São Paulo.

A amostra consistiu de 22 publicações, sendo duas dissertações, uma tese e 19 artigos científicos que incluem os ter mos comunicação, enfermagem, gerenciamento, gestão ou administração. D os 19 artigos, 18 são da base de dados L I L ACS e um é da base M E D L I NE . A s duas disser tações e a tese são do banco de dados DEDALUS.

Os dados foram registrados em um formulário para coleta de dados bibliográficos. D entre as várias técnicas propostas para análise dos dados, optou-se pela utilização da análise de conteúdo ${ }^{(13)}$. Procedeu-se a leitura exaustiva dos artigos com posterior categorização por conteúdo temático. Os estudos foram agrupados em categorias a partir das conexões e relações apresentadas pelos mesmos, de for ma que possibilitassem explicações einterpretações do tema investigado.

\section{RESULTADOS E DISCUSSÃO}

$\mathrm{Na}$ apresentação dos resultados serão destacados dados referentes aos autores, ao tipo de publicação, às bases de dados utilizadas, bem como dados referentes ao tipo de abordagem metodológica. A pós, serão apresentadas as categorias do estudo descritas no Quadro 1 a seguir.

\begin{tabular}{|c|c|}
\hline \multirow{6}{*}{$\begin{array}{l}\text { Comunicação como forma } \\
\text { de se alcançar a eficiência } \\
\text { organizacional }\end{array}$} & $\begin{array}{l}\text { Pereira M CA, Fávero N. G erenciamento: a comunicação na dinâmica } \\
\text { motivacional do trabal ho da equipe de enfermagem; } 2000^{(14)} \text {. }\end{array}$ \\
\hline & $\begin{array}{l}\text { Peres A M , Ciampone M H T. G erência e competências gerais do enfer- } \\
\text { meiro; } 2006^{(15)} \text {. }\end{array}$ \\
\hline & $\begin{array}{l}\text { Campos LF, M elo M R AC. Os desafios da comunicação administrativa } \\
\text { na enfermagem; } 2002^{(16)} \text {. }\end{array}$ \\
\hline & $\begin{array}{l}\text { A breu LO, M unari DB, Queiroz ALB, Fernandes CNS. } 0 \text { trabalho de } \\
\text { equipe em enfermagem: revisão sistemática da literatura; } 2005^{(17)} \text {. }\end{array}$ \\
\hline & $\begin{array}{l}\text { Silva RF, Tanaka OY. T écnica D elphi: identificando as competências } \\
\text { gerais do médico e do enfermeiro que atuam em atenção primária de } \\
\text { saúde; } 1999^{(18)} \text {. }\end{array}$ \\
\hline & $\begin{array}{l}\text { Sanna M C. A evolução da disciplina de administração aplicada à enfer- } \\
\text { magem na escola de enfermagem da U SP no período de } 1980 \text { a 1995; } \\
\text { 1999(19). }\end{array}$ \\
\hline
\end{tabular}


Continuação.

\begin{tabular}{|c|c|}
\hline & $\begin{array}{l}\text { Spagnol CA, Ferraz, CA. T endências e per spectivas da administração } \\
\text { em enfermagem: um estudo na Santa Casa de Belo Horizonte-M G; } \\
2002^{(20)} \text {. } \\
\text { N eto D L. Realidade I nter acionista da comunicação na gerência em en- } \\
\text { fermagem: significados, ações e mudanças; } 2002^{(21)} \text {. }\end{array}$ \\
\hline $\begin{array}{l}\text { Comunicação e sua relação } \\
\text { com a liderança do enfer meiro } \\
\text { e a tomada de decisão. }\end{array}$ & $\begin{array}{l}\text { Fernandes M S, Spagnol CA, T revizan M A, H ayashida M . A conduta } \\
\text { gerencial da enfermeira: um estudo fundamentado nas teorias gerais } \\
\text { da administração; } 2003^{(22)} \text {. } \\
\text { T revizan M A, M endes IAC, F ávero N, M el o M RAC. Liderança e co- } \\
\text { municação no cenário da gestão em enfermagem; } 1998^{(23)} \text {. } \\
\text { G alvão CM , Sawada N O, Castro AP, Corniani F. L iderança e Comuni- } \\
\text { cação: estratégias essenciais para o gerenciamento da assistência de en- } \\
\text { fermagem no contexto hospitalar; } 2000^{(2)} \text {. } \\
\text { Spagnuolo R S, Pereira M LT. Práticas de saúde em E nfermagem e Co- } \\
\text { municação: um estudo de revisão da literatura; } 2007^{(24)} \text {. } \\
\text { Berto GS, Cunha KC. A participação do enfermeiro no processo decisório; } \\
2000^{(25) .}\end{array}$ \\
\hline $\begin{array}{l}\text { Comunicação entre os membros } \\
\text { da equipe de enfermagem } \\
\text { durante passagem de plantão } \\
\text { e reuniões de equipe. }\end{array}$ & $\begin{array}{l}\text { A ndrade JS, V ieira M J, Santana M A, Lima D M . A comunicação entre } \\
\text { enfermeiros na passagem de plantão; } 2004^{(26)} \text {. } \\
\text { Siqueira I LCP, Kurcgant P. Passagem de plantão: fal ando de paradigmas } \\
\text { e estratégias; } 2005^{(27)} \text {. } \\
\text { Gomes E S, A nselmo M E O, Filho W D L. As reuniões de equipe como } \\
\text { elemento fundamental na organização do trabal ho; } 2000^{(28)} \text {. }\end{array}$ \\
\hline $\begin{array}{l}\text { Comunicação como fator gerador } \\
\text { de satisfação e insatisfação no } \\
\text { trabalho de enfermagem. }\end{array}$ & $\begin{array}{l}\text { Santos M S. A (in)satisfação do enfermeiro no trabalho: implicações } \\
\text { para o ger enciamento das ações de enfermagem: aspectos teóricos; } \\
1999^{(29)} \text {. }\end{array}$ \\
\hline $\begin{array}{l}\text { Comunicação como forma de lidar } \\
\text { com conflitos. }\end{array}$ & $\begin{array}{l}\text { A gostini R. O conflito como fenômeno organizacional: identificação e } \\
\text { abordagem na equipe de enfermagem de um hospital público; } 2005^{(30)} \text {. } \\
\text { M endes E M T, M ayor E RC, Francisco M CPB, Silva M JP, Capeli SCA. } \\
\text { Revendo estruturas e reorganizando nossa comunicação, 2000 (31). }\end{array}$ \\
\hline $\begin{array}{l}\text { Comunicação no gerenciamento } \\
\text { de centro cirúrgico e na central de } \\
\text { material e esterilização }\end{array}$ & $\begin{array}{l}\text { Stumm EM F, M acalai RT, Kirchner RM . Dificuldades enfrentadas por } \\
\text { enfermeiros em um centro cirúrgico; } 2006^{(32)} \text {. } \\
\text { T aube SAM , M eier M J. O processo de trabalho da enfermeira na cen- } \\
\text { tral de material e esterilização; } 2007^{(33)} \text {. }\end{array}$ \\
\hline $\begin{array}{l}\text { Comunicação do enfer meiro no } \\
\text { H ome Care. }\end{array}$ & $\begin{array}{l}\text { Alves M , A raújo M T, Santana D M , V ieira D L. T rabal ho do enfer meiro } \\
\text { em uma empresa de H ome Care de B elo H orizonte, Brasil; } 2007^{(34)} \text {. }\end{array}$ \\
\hline
\end{tabular}

Q uadro 1 - Sistematização dos artigos / dissertações / tese encontrados sobre comunicação em enfermagem e gerenciamento, segundo a categoria a que pertencem. Ribeirão Preto, SP, 2009. 
No que tange à categoria profissional, a que mais se destacou em número de publicação de artigos e dissertações que tratam sobre o tema comunicação em enfermagem e ger enciamento foi a de enfermeiros (95,45\%), sendo que a maioria dos primeiros autores possui titulação de mestre (50\%), seguido dos doutores ${ }^{(27,29)}$. 0 tipo de publicação mais encontrado sobre a temática pesquisada foi na forma de artigos (86,4\%) que foram publicados em oito periódicos, sendo que a Revista L atino-A mericana de E nfermagem foi a que mais se destacou (21\%).

A grande maioria das publicações foi acessada na íntegra pelo Portal Scielo (68,2\%), que se trata de uma biblioteca científica virtual confiável que abrange uma coleção sel ecionada de periódicos científicos brasileiros. 0 método descritivo foi o mais utilizado pelos autores $(36,4 \%)$ e a abordagem qualitativa foi a de maior destaque $(54,5 \%)$.

Com a finalidade de facilitar a interpretação dos dados, as publicações foram ag rupadas em sete categorias.

$\mathrm{Na}$ categoria "Comunicação como forma de se alcançar a eficiência organizacional" se destaca a importância da val orização da comunicação enquanto instrumento para 0 alcance de êxito nas mudanças atuais propostas para a organização do trabalho.

Os artigos / tese descrevem que a comunicação é el eita como uma das competências necessárias para que toda equipe multiprofissional atue com responsabilidade e eficiência na atenção à saúde, tornando assim as instituições organizadas e com qualidade no atendimento.

D estaca-se a importância da comunicação no trabal ho da equipe de enfermagem, já que o funcionamento eficiente desta equipe, somado aos trabalhos necessários das outras categorias profissionais, garante um atendimento adequado em saúde. É esclarecido, porém, que o processo comunicativo deve ser claro, a fim de integrar a equipe de enfermagem e conseguir, desta forma, a busca de objetivos e metas comuns da equipe.

Alguns estudos abordam o processo comunicativo como ferramenta do gerenciamento, apontando a necessidade e a essencial idade da comunicação como estratégia para garantia do sucesso do gerenciamento da assistência de enfermagem. Reiteram ainda que a reestruturação administrativa que desencadeou um modelo de gestão compartiIhado ou colegiado levou à valorização do trabalho em equipe e das relações interpessoais. 0 processo comunicativo favoreceu um diálogo efetivo entre diferentes profissionais da equipe multiprofissional e de enfer magem, e desta forma, o processo decisório tornou-se descentralizado(5,19)

Desta forma, os artigos convergem para a idéia de que a comunicação das equipes de saúde atua como fator de ag regação ou desagregação da organização do trabalho em saúde.

Os artigos agrupados na categoria "Comunicação e sua relação com a liderança do enfermeiro e a tomada de decisão" remetem à reflexão de que a comunicação é uma habilidade que deve ser desenvolvida por enfermeiros responsáveis por liderarem equipes de enfermagem, uma vez que ela propicia o trabal ho em equipe que aumenta a possibilidade da prestação de uma assistência livre de riscos aos pacientes.

D esta forma, através da adequação da comunicação e da liderança, o enfer meiro pode implementar mudanças e, através delas, dividir idéias e informações com sua equipe, a fim de desenvolver habilidades e promover maturidade nos liderados, compartilhando das decisões relativas à prática profissional $^{(24)}$.

A comunicação é tida como elemento componente da prática de lider ança que deve ser exercida pelo enfermeiro, sendo essencial para a influência e coordenação de atividades, desde que o comunicador possua habilidade para transmitir informações de for ma que el a seja recebida sem distorções.

$\mathrm{Na}$ categoria "Comunicação entre os membros da equipe de enfermagem durante passagem de plantão e reuniões de equipe" os artigos explicitam a importância da troca de informações, principalmente por meio da comunicação verbal, para garantir qualidade à passagem de plantão e continuidade da assistência prestada ao cliente.

A comunicação verbal escrita também é necessária, já que é uma ferramenta importante de sustentação do processo de cuidar. Porém, os profissionais devem se atentar para a anotação correta e a qualidade das mesmas ${ }^{(26,27)}$, uma vez que trata-se de instrumento legal usado como fonte de informação clínica e administrativa, além de respaldar os profissionais em relação a eventuais questionamentos jurídicos e processuais.

D estaca-se também a importância das reuniões frequentes da equipe de enfermagem, como estratégia para garantir o bom andamento do trabalho ${ }^{(28)}$. Essas reuniões devem configurar-se em espaços de análise e discussão do processo de tra- 
balho que, consequentemente podem colaborar com a qualificação da assistência prestada.

D esta maneira, a comunicação, seja ela verbal escrita ou falada, quando se dá de maneira satisfatória, contribui para a fluência do atendimento de enfermagem e, consequentemente, para 0 desempenho gerencial eficaz.

A categoria "Comunicação como fator gerador de satisfação e insatisfação no trabalho de enfermagem" relaciona a comunicação com os fatores geradores de (in)satisfação no trabalho de enfer magem, bem como com as implicações para 0 gerenciamento das ações de enfermagem ${ }^{(29)}$. "É altamente desejável que as instituições de saúde valorizem o processo comunicativo, uma vez que 0 desenvolvimento de habilidades específicas nesse sentido pode proporcionar maior satisfação e motivação no trabalho. A pouca comunicação ou até mesmo a falta dela entre a equipe de enfer magem é uma das razões apontadas pel os enfer meiros como fatores geradores de insatisfação no ambiente de trabal ho"(29).

E $m$ relação à categoria "Comunicação como forma de lidar com conflitos" al guns estudos trazem que no M odelo Colegiado ou Compartilhado de G estão podem emergir conflitos, uma vez que pressupõe a descentralização do poder e a participação do coletivo nas decisões( ${ }^{(5)}$. N este caso, o profissional que ocupa posição de articulador ou gestor da equipe deve ter habilidades comunicativas, incentivando o diálogo e a conseqüente superação do conflito tanto na interação de grupos profissionais como nas relações humanas com o paciente ${ }^{(30)}$.

A categoria "Comunicação no gerenciamento de Centro Cirúrgico e na Central de Material e Esterilização" trata da comunicação entre os profissionais e o relacionamento interpessoal como fortes fatores de sucesso para gerenciar e organizar o Centro Cirúrgico e a Central de M aterial e Esterilização.

A comunicação eficaz estabelece teias de relações grupais e fortalece a relação entre os profissionais já que, por meio dela, a troca de opiniões e idéias contribui ativamente para a convivência das equipes e o sucesso do trabal ho ${ }^{(31,32)}$.

Contudo, quando a comunicação multiprofissional apresenta dificuldades, o enfermeiro é um dos profissionais que reconhece isto e, busca solucionar os problemas, reconhecendo tal dificuldade e atribuindo meios de estabelecer formas de entendimento(32).
E m muitas situações, o enfer meiro assume 0 papel de ger ente das relações interpessoais, e para que haja sucesso, a comunicação tor na-se um veículo essencial(31), já que é um instrumento básico, uma ferramenta do processo de trabalho do enfermeiro, com a qual se pode transformar a consciência individual e coletiva, articular teoria e prática e qualificar as ações da equipe de enfermagem.

O utra categoria encontrada refere-se à "Comunicação do enfermeiro no Home Care". A comunicação aberta, clara e eficiente no H ome Careé tão necessária quanto em uma instituição hospitalar, especialmente no que tange ao estabelecimento de uma relação harmônica entre o cuidador, 0 paciente e sua família.

T anto a comunicação como a postura profissional do enfermeiro são importantes para a relação com seus pacientes neste serviço, já que são essenciais para a aceitação dos cuidadores e do próprio paciente $\mathrm{e}^{(33)}$.

0 enfermeiro deve ainda ser comunicativo com a equipe multidisciplinar, visando circular informações a respeito do paciente, tanto por meio da comunicação verbal falada, como pela transmissão de relatórios intra e inter equipes, a fim de assegurar com qualidade a ação ter apêutica.

\section{CONSIDERAÇÕES FINAIS}

A través da leitura das publicações encontradas percebe-se que a maioria dos autores cita o processo comunicativo como um dos pontos necessários para se al cançar o êxito gerencial em instituições de saúde, salientando as várias contribuições que a comunicação clara e adequada é capaz de propiciar às equipes.

Para atingir o sucesso organizacional nas instituições, o enfermeiro deve ser o el o da cadeia comunicativa, uma vez que está constantemente em contato com a equipe multiprofissional. Além disso, a insuficiência do processo de comunicação é responsável pelo desencadeamento de fatores geradores de insatisfação nas instituições de saúde.

Quando o modelo gerencial da instituição é pautado na A bordagem Clássica da Administração a comunicação vertical izada, truncada e, portanto, ineficiente. Já quando se adota o estilo contemporâneo de gestão, busca-se a intensificação da comunicação em todos os níveis, ou seja, vertical e horizontalmente. 
A comunicação ineficiente entre a equipe de enfermagem, seja durante a passagem de plantão ou em qualquer outra situação no desenvolvimento do trabalho, pode acarretar em má qualidade na assistência prestada. D essa forma, a comunicação é fator de interferência na dinâmica de funcionamento de qualquer instituição de saúde, sendo fundamental para o desenvolvimento do trabal ho.

D esta forma, reitera-se que todas as publicações do presente estudo, discorrem sobre a importância do desenvolvimento de habilidades comunicativas como meio de se atingir a eficácia e o sucesso na dinâmica de trabal ho organizacional.

$\mathrm{N}$ ota-se que tais estudos convergem para a idéia da importância do papel comunicativo para a vida profissional dos trabalhadores de saúde, em especial dos enfermeiros. F ica claro que a comunicação apresenta-se como uma necessidade no contexto da administração de enfermagem. A eficácia, a rapidez e a atual ização da comunicação entre os profissionais de enfermagem influenciam vários aspectos necessários da conduta profissional dos enfermeiros, como o exercício da liderança, a tomada de decisões e o planejamento da assistência de enfermagem.

A ssim, as publicações encontradas confirmam que a comunicação na administração em enfermagem constitui uma fer ramenta essencial para o sucesso do desempenho profissional, tornando-se assim uma área ampla de pesquisa que possibilita inúmeras explorações.

\section{REFERÊ NCIAS}

1 Kurcgant P, Cunha KC, M assarollo M CKB, Ciampone M HT, Silva VE F, Castilho V, et al. A dministração em enfermagem. São Paulo: EPU ; 1991.

2 G alvão CM , Sawada N O, Castro AP, Corniani F. Liderança e comunicação: estratégias essenciais para 0 gerenciamento da assistência de enfer magem no contexto hospitalar. Rev Latino-A m E nfermagem. 2000; 8(5):34-43.

3 W erlang SC, Azzolin K, M oraes M A, Souza E N. Comunicação não verbal do paciente submetido à cirurgia cardíaca: do acordar da anestesia à extubação. R ev G aúcha Enferm. 2008;29(4):551-6.

4 Ferraz CA, Valle ERM . Administração em enfermagem: da gerência científica à gerência sensível. I n: Organización Panamericana de la Salud. La enfermeria em las A méricas. W ashington (DC); 1999. p. 205-26.
5 Spagnol CA. Tendência e perspectives da administração em enfermagem: um estudo na Santa Casa de Belo H orizonte [ dissertação] . Ribeirão Preto: E scola de Enfermagem de Ribeirão Preto, U niversidade de São Paulo; 2000.

6 M oraes AM P. I niciação ao estudo da administração. 2a ed. São Paulo: Pearson Education do Brasil; 2001.

7 Bernardes A . G estão colegiada: a visão da equipe multiprofissional [ tese] . Ribeirão Preto: Escola de Enfermagem de Ribeirão Preto, U niversidade de São PauIo; 2005.

8 Bernardes A, Cecílio LCO, N akao JRS, Évora Y D M . Os ruídos encontrados na construção de um modelo democrático e participativo de gestão hospitalar. Ciênc Saúde Colet. 2007;12(4):861-70.

9 Bernardes A, Évora Y DM, N akao JRS. G estão Colegiada na visão dos técnicos e auxiliares de enfermagem em um hospital público brasileiro. Ciênc Enferm. 2008;14(2):65-74.

10 M otta PR . G estão contemporânea: a ciência e a arte de ser dirigente. 13a ed. Rio de Janeiro: Record; 2002.

$11 \mathrm{G}$ anong $\mathrm{LH}$. Integ rative reviews of nursing research. Res N urs H ealth. 1987;10(1):1-11.

12 Polit DF, Beck CT, Hungler BP. Fundamentos de pesquisa em enfer magem: métodos, aval iação eutilização. 5ạ ed. Porto Alegre: Artmed; 2004.

13 M inayo M CS. 0 desafio do conhecimento: pesquisa qualitativa em saúde. São Paulo: Hucitec; 2000.

14 Pereira M CA, F ávero N, T revizan M A. Gerenciamento: a comunicação na dinâmica motivacional do trabal ho da equipe de enfermagem. In: A nais do 70 Simpósio Brasileiro de Comunicação em Enfermagem; 2000 jun 5-6; R ibeirão Preto, Brasil. R ibeirão P reto: E scola de E nfermagem de Ribeirão Preto, U niversidade de São Paulo; 2000. p. 102.

15 Peres A M , Ciampone M HT. G erência e competências gerais do enfermeiro. Texto Contexto $\mathrm{E}$ nferm. 2006;15(3):492-9.

16 Campos LF, M elo M RAC. Os desafios da comunicação administrativa na enfermagem. In: A nais do 8- Simpósio Brasileiro de Comunicação em Enfermagem; 2002 maio 2-3; Ribeirão Preto, Brasil. Ribeirão Preto: E scola deE nfermagem de Ribeirão Preto, U niversidade de São Paulo, 2002. p. 124. 
17 A breu LO, M unari DB, Queiroz ALB, Fernandes CNS. 0 trabal ho de equipe em enfer magem: revisão sistemática da literatura. Rev Bras Enferm. 2005; 58(2):203-7.

18 Silva RF, T anaka OY. T écnica D el phi: identificando as competências gerais do médico e do enfermeiro que atuam em atenção primária de saúde. Rev Esc Enferm U SP. 1999;33(3):207-16.

19 Sanna M C. A evolução da disciplina de administração aplicada à enfer magem na escola de enfermagem da U SP no período de 1980 a 1995. Rev E sc Enferm USP. 1999;33(1):17-30.

20 Spagnol CA, Ferraz CA. Tendências e perspectivas da administração em enfer magem: um estudo na Santa Casa de Belo H orizonte-M G. Rev Latino-A m E nfermagem. 2002;10(1):15-20.

21 Neto D L. Realidade interacionista da comunicação na gerência em enfer magem: significados, ações e mudanças [ tese] . Fortaleza: F aculdade de F armácia, Odontologia e Enfermagem, U niversidade Federal do Ceará; 2002.

22 Fernandes M S, Spagnol CA, T revizan M A, H ayashida M . A conduta gerencial da enfer meira: um estudo fundamentado nas teorias gerais da administração. Rev Latino-Am E nfermagem. 2003;2(11):161-7.

23 T revizan M A, M endes I AC, F ávero N, M elo M RAC. Liderança e comunicação no cenário da gestão em enfermagem. Rev Latino-Am Enfermagem. 1998; 6(5):77-82.

24 Spagnuolo RS, Pereira M LT. Práticas de saúde em enfermagem e comunicação: um estudo de revisão da literatura. Ciênc Saúde Colet. 2007;12(6):1603-10.

25 Berto GS, Cunha KC. A participação do enfer meiro no processo decisório. T exto Contexto E nferm. 2000; 9(2 Pt 2):737-51.
26 Andrade JS, Vieira M J, Santana M A, Lima D M . A comunicação entre enfermeiros na passagem de plantão. A cta Paul Enferm. 2004;17(3):311-5.

27 Siqueira I LCP, Kurcgant P. Passagem de plantão: falando de paradigmas e estratégias. Acta Paul E nferm. 2005;18(4):446-51.

$28 \mathrm{G}$ omes ES, A nselmo M E O, F ilho W D L. As reuniões de equipe como el emento fundamental na organização do trabalho. Rev Bras Enferm. 2000;53(3):47280.

29 Santos M S. A (in)satisfação do enfer meiro no trabaIho: implicações para o gerenciamento das ações de enfermagem: aspectos teóricos [ disser tação] . Ribeirão Preto: Escola de Enfermagem de Ribeirão Preto, U niversidade de São Paulo; 1999.

30 Agostini R. 0 conflito como fenômeno organizacional: identificação e abordagem na equipe de enfer magem de um hospital público [ dissertação] . R ibeirão Preto: Escola de Enfermagem de Ribeirão Preto, U niver sidade de São Paulo; 2005.

31 M endes EM T, M ayor ERC, Francisco M CPB, Silva M JP, Capeli SCA. Revendo estruturas e reorganizando nossa comunicação. Rev Bras Enferm. 2000;53 (3):450-7.

32 Stumm EM F, M acalai RT, Kirchner RM. Dificuldades enfrentadas por enfermeiros em um centro cirúrgico. T exto Contexto Enferm. 2006;15(3):46471.

33 T aube SAM, M eier MJ. 0 processo de trabalho da enfermeira na central de material e esterilização. Acta Paul Enferm. 2007;20(4):470-5.

34 A Ives M , A raúijo M T, Santana D M , Vieira D L . T rabal ho do enfermeiro em uma empresa de $\mathrm{H}$ ome Care de Belo H orizonte, Brasil. I nvest E duc E nferm. 2007; 2(25):96-106.

Recebido em: 18/ 12/ 2009

A provado em: 15/ 05/ 2010

\section{Endereço da autora / Dirección del autor / Author's address:}

Andrea Bernardes

Av. Bandeirantes, 3900, Campus da U SP

14040-902, Ribeirão Preto, SP

E-mail: andreab@eerp.usp.br 